\title{
Bioanalysis
}

\section{Squeezing more value from the analytes we have: personal baselines for multiple analytes in serial DBS}

\author{
"Adoption of new protein biomarkers continues to be very slow, \\ adding approximately 1.5 new proteins per year to the 115 so far \\ approved by the US FDA..."
}

First draft submitted: 5 April 2016; Accepted for publication: 13 May 2016; Published online: 9 June 2016

\section{Keywords: DBS $\bullet$ MS $\bullet$ panels $\bullet$ personalized}

Combining high-throughput multiplex protein assays based on immunoaffinity MS (SISCAPA) with patient-friendly longitudinal sample collection using finger-prick DBS technology enables the creation of a personalized longitudinal baseline and $\mathrm{CV}$ for each assay. The increases in statistical power attributable to use of panels (instead of single analyte tests) and personalized test interpretation (instead of conventional population reference intervals) offers a means to extract significant increases in clinical diagnostic value from the menu of known clinical protein analytes.

Adoption of new protein biomarkers continues to be very slow, adding approximately 1.5 new proteins per year to the 115 so far approved by the US FDA as useful analytes for in vitro diagnostic (IVD) tests in plasma or serum [1]. Considerable discussion has focused on potential reasons for this disappointing performance, including inadequate research investment, regulatory and/or physician resistance and the limitations imposed by biology itself. We believe that biological complexity may be the major limitation in particular the scope of normal variations between individuals and within individuals over time, as well as the variety of disease mechanisms affecting them. If this is the case, then major improvements in diagnostics might be achieved by using the existing clinical analytes more effectively: in panels covering multiple aspects of a disease pro- cess, interpreted against personal baselines instead of currently used population reference intervals. Synergistic advances in sample collection (e.g., DBS), multiplex testing technology (e.g., MS) and bioinformatics (i.e, 'big data') provide a basis for practical considerations of this approach.

\section{Tests using panels versus single biomarkers}

Almost all clinically used protein biomarkers are currently measured by individual immunoassays on separate sample aliquots, an approach that entails penalties in terms of cost and sample consumption for each additional analyte measured. Thus, if an additional biomarker contributes less than a startling increase in predictive power, or even if it provides great power but only in a subpopulation of patients, today it is unlikely to be measured routinely. Nevertheless, many disease states, including most chronic conditions, affect multiple disease biomarkers. Numerous proteins, including CRP, apolipoproteins A-I, B, C-III and (a), MPO and Lp-PLA2, contribute independent information on cardiovascular risk, for example. This should not be surprising, given the complexity of the disease processes involved. Combinations of such well-studied markers, many with established mechanistic roles, provide a powerful alternative to de novo development of candidate lists emerging from small-scale unbiased proteome screens, but such com-

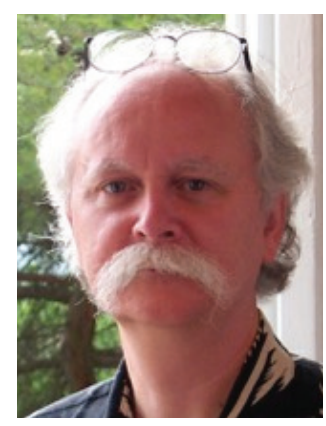

Leigh Anderson

Author for correspondence: SISCAPA Assay Technologies, Washington DC USA \& Victoria BC, Canada

leighanderson@siscapa.com

\section{Morteza Razavi}

SISCAPA Assay Technologies, Washington DC USA \& Victoria BC, Canada

\section{Steven Skates}

Massachusetts General Hospital \& Harvard Medical School, Boston, MA, USA

\section{Norman G Anderson}

Viral Defense Foundation, Rockville, MD, USA

Terry W Pearson

SISCAPA Assay Technologies, Washington DC USA \& Victoria BC, Canada

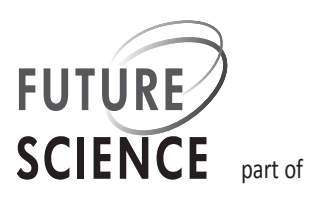


binations are rarely used clinically because of cost considerations.

As a method of protein measurement, MS changes the equation for multiplex panels: one aliquot of sample is processed to generate the first protein value, but numerous additional proteins can be measured in the same operation at relatively low incremental cost above that of the single-analyte workflow. Such methods are becoming practical in the clinical laboratory as demonstrated in the recent paper by van den Broeck et al. [2], which describes the measurement of apolipoproteins A-I, B, C-I, C-II, C-III and E in a single MS assay, with performance equivalent to conventional automated, single biomarker immunoassays. The sensitivity and throughput of multiplex MS assays can be further improved using analyte enrichment methods such as SISCAPA [3], which overcome the shortcomings of immunoassay technologies.

\section{"However, several lines of evidence make it clear that this 'one size fits all' approach is an imprecise view of the human population and does not accurately reflect disease biology."}

\section{Personal baselines versus population reference intervals}

Almost all clinical test results are interpreted in terms of population reference intervals, an approach that assumes similar levels and similar change behavior for disease biomarkers in all individuals. This is a major convenience for physicians in spotting out-of-range values, and for laboratorians who can establish a local reference interval from a few hundred 'normal' samples. However, several lines of evidence make it clear that this 'one size fits all' approach is an imprecise view of the human population and does not accurately reflect disease biology.

The data assembled by Ricos et al. [4] on betweensubject and within-subject variation in laboratory test values demonstrates that many widely used analytes vary much less in a normal person over time than between people in the population. This means that personally significant biomarker changes are easily buried in the between-subject variation of the population (i.e., by interpretation against the population reference interval), effectively discarding small but statistically significant changes from a subject's normal baseline that can provide early signals of disease. In some situations, using the population variation can degrade the performance of a biomarker so as to make it appear useless, whereas interpretation against a baseline of the patient's own predisease measurements identifies clinically significant changes. A striking example of the value of longitudinal measurement is presented in a recent paper in which the use of the 'risk of ovarian cancer algorithm' (ROCA) on serial CA-125 measurements 'doubled the number of screen-detected primary invasive epithelial ovarian or tubal cancers compared with a fixed cutoff at the time of detection by ROCA' [5]. Similarly, the use of the Relative Change Value (RCV) approach to evaluate each patient against himself or herself substantially improved performance of alpha-fetoprotein (AFP) in screening for hepatocellular carcinoma [6]. In fact, some form of longitudinal interpretation is likely to be superior for all protein biomarkers for which the variation within the individual $\left(\mathrm{CV}_{\mathrm{I}}\right)$ over time is less than 0.6times the variation between individuals $\left(\mathrm{CV}_{\mathrm{G}} ;[7]\right)$, that is, for CA-125, AFP and, astonishingly, a total of $69 \%$ of the existing clinical serum and plasma protein test menu covered by Ricos' data.

Taking account of biological variation is certainly not a new concept in laboratory medicine, and it has been carefully explained to a broad audience repeatedly, for example, by Callum Fraser in his AACC Press book 'Biological Variation: From Principles to Practice' [7]. Nevertheless, despite abundant reasons to use the approach for many if not most biomarkers, personal baselines or personalized algorithms are not routinely available, perhaps because of insufficient method standardization across laboratories, or due to the difficulty of assembling usable longitudinal baseline measurements from existing medical records.

In its purest form, measurement of 'normal' personal baselines, together with 'normal' personal standard deviations from that baseline, allow biomarker data to be presented in terms of statistical significance for the individual: how many personal standard deviations away from a personal baseline is a given laboratory result? Such an approach (described in the accompanying paper in this issue [8]) significantly increases existing biomarkers' clinical utility through detection of changes occurring over time. Similar approaches such as ROCA also yield increased clinical utility while providing the same utility for diagnosis from a single time-point sample as current methods for interpreting biomarkers.

\section{DBS versus blood drawn by phlebotomy}

Routine periodic collection of blood samples to support personalized test interpretation is currently considered impractical because of cost and patient inconvenience. The cost of phlebotomy, in terms of personnel, materials, institutional infrastructure and transportation (e.g., via FedEx) is substantial, and rarely reimbursable without an immediate diagnostic need. Patients are likewise not enthusiastic about recurring schedule interruptions, travel to a phlebotomy center and negative visceral reactions to the venipuncture itself. 
An alternative sampling technology, based on Guthrie's implementation [9] of DBS on filter paper for newborn screening, has been explored in a variety of contexts for a decade or more. Numerous publications have confirmed that a wide array of metabolites, drugs and proteins can be measured in such samples [10,11], and that individuals can perform effective finger prick sample collection at home [12]. DBS samples are not fully equivalent to conventional venipuncture specimens in terms of accurately known plasma volume or concentration of some biomarkers (e.g., proteins elevated in interstitial fluid compared with venous blood), but these limitations can be largely overcome using new MS-based methods. Indeed the ability of multiplex MS technologies to measure additional 'normalizing' analytes (e.g., albumin, other major plasma proteins, or hemoglobin) at low cost facilitates normalization of punch volume and hematocrit variations in DBS. Even where such corrections do not restore fingerprick values to plasma blood draw values, the use of personal baselines may restore clinical utility for analytes that show plasma:fingerprick differences [8]. Along with their ease of collection, low cost and demonstrated suitability for multiplexing, DBS are well suited for longitudinal sampling and determination of practical personal baselines, providing an attractive option for monitoring wellness, early detection of disease and monitoring of chronic conditions.

\section{An emerging solution for personalized diagnostics?}

Our purpose here is to draw attention to the synergy among the three strands of technology outlined

\section{References}

Papers of special note have been highlighted as: $\bullet$ of interest

1 Anderson NL, Ptolemy AS, Rifai N. The riddle of protein diagnostics: future bleak or bright? Clin. Chem. 59, 194-197 (2013).

2 van den Broek I, Romijn FP, Nouta J et al. Automated multiplex LC-MS/MS assay for quantifying serum apolipoproteins A-I, B, C-I,C-II, C-III, and E with qualitative apolipoprotein E phenotyping. Clin. Chem. 62(1), 188-197 (2015).

3 Razavi M, Anderson NL, Pope ME, Yip R, Pearson TW. High precision quantification of human plasma proteins using the automated SISCAPA Immuno-MS workflow. N. Biotechnol. doi:10.1016/j.nbt.2015.12.008 (2016) (Epub ahead of print).

4 Ricos C, Alvarez V, Cava F. Current databases on biological variation: pros, cons and progress. Scand. J. Clin. Lab. Invest. 59, 491-500 (1999).

- Updated on the website at: www.westgard.com/biodatabase1.htm above, and the resulting opportunity to increase the value of the menu of known protein biomarkers in pharmaceutical development and laboratory diagnostic testing. MS makes it possible to measure numerous clinically important proteins in DBS samples that can be collected periodically by individuals at home (or anywhere) and sent for analysis to a central laboratory by regular mail. Emerging platforms for mobile (mHealth) or electronic (eHealth) personal and medical information enormously increase our capacity to collect, store and digest a history of quantitative biomarker measurements, and with it to provide valuable feedback (and education) to an enthusiastic user base. This longitudinal approach provides a new and powerful means of squeezing substantially more value from the biomarkers we have, as well as a superior method for validating and translating novel protein biomarkers, addressing unmet medical needs such as Alzheimer's disease, COPD and cancer.

\section{Financial \& competing interests disclosure}

N Leigh Anderson, M Razavi and TW Pearson are supported by SISCAPA Assay Technologies, Inc.; SJ Skates is supported by Grant No. CA152990 from the National Cancer Institute Early Detection Research Network. The authors have no other relevant affiliations or financial involvement with any organization or entity with a financial interest in or financial conflict with the subject matter or materials discussed in the manuscript apart from those disclosed.

No writing assistance was utilized in the production of this manuscript.

5 Menon U, Ryan A, Kalsi J et al. Risk algorithm using serial biomarker measurements doubles the number of screendetected cancers compared with a single-threshold rule in the United Kingdom collaborative trial of ovarian cancer screening. J. Clin. Oncol. 33(18), 2062-2071 (2015).

6 Trapé J, Botargues JM, Porta F et al. Reference change value for alpha-fetoprotein and its application in early detection of hepatocellular carcinoma in patients with hepatic disease. Clin. Chem. 49, 1209-1211 (2003).

7 Fraser CG. Biological Variation: From Principles to Practice. AACC Press, Washington, DC, 151 (2001).

8 Razavi M, Anderson NL, Yip R, Pope ME, Pearson TW. Multiplexed longitudinal measurement of protein biomarkers in dried blood spots using an automated SISCAPA workflow. Bioanalysis 8(15) 1597-1609(2016).

9 Guthrie R, Susi A. A simple phenylalanine method for detecting phenylketonuria in large populations of newborn infants. Pediatrics 32, 338-343 (1963).

10 Lehmann S, Delaby C, Vialaret J, Ducos J, Hirtz C. Current and future use of 'dried blood spot' analyses in clinical chemistry. Clin. Chem. Lab. Med. 51, 1-13 (2015). 
11 Chambers AG, Percy AJ, Yang J, Camenzind AG, Borchers $\mathrm{C} \mathrm{H}$. Multiplexed quantitation of endogenous proteins in dried blood spots by multiple reaction monitoring mass spectrometry. Mol. Cell. Proteom. 12(3), 781-791 (2012).
12 Tanna S, Lawson G. Self-sampling and quantitative analysis of DBS: can it shift the balance in over-burdened healthcare systems? Bioanalysis 7, 1963-1966 (2015). 